\title{
Realidade Aumentada na Educação: Uma Análise das Ferramentas Flaras e Aumentaty como Recursos para Aulas Expositivas
}

\author{
David Severo do Nascimento Júnior - UFPB/UEPB - davidsnjr@ hotmail.com \\ Ayla Débora Dantas de Souza Rebouças - UFPB - ayla@dcx.ufpb.br
}

\begin{abstract}
Resumo: O ensino apresenta-se como parte indissociável da educação formal, portanto, é uma atividade que requer um interesse legítimo na busca constante por novas práticas pedagógicas. Neste sentido, cabe ao educador o desafio de utilizar dispositivos inovadores que sejam capazes de atrair a atenção dos estudantes, tarefa cada vez mais complexa, principalmente na sociedade atual, cuja tecnologia coloca-se como eixo central. Diante de uma geração que vivencia uma "revolução tecnológica" bastante consolidada, a Realidade Aumentada (RA) apresenta-se como instrumento pertinente, no sentido de promover a inclusão de elementos lúdicos durante o processo de aprendizagem. O objetivo principal deste artigo é avaliar a viabilidade do uso de ferramentas de RA como o Flaras e o Aumentaty como recursos para aulas expositivas considerando sua aceitação por professores. Após este trabalho, foram obtidos indícios de que ferramentas de RA podem ser utilizadas por qualquer docente que deseje explorá-las em sala e aprender sobre elas desde que receba o apoio necessário.
\end{abstract}

Palavras-chave: realidade aumentada, ensino, Aumentaty.

\section{Augmented Reality in Education: An Analysis of the Flaras and Aumentaty Tools as Resources for Lectures}

\begin{abstract}
Education is an inseparable part of formal education, so it is an activity that requires a legitimate interest in the constant search for new pedagogical practices. In this sense, it is up to the educator the challenge to use innovative devices that are capable of attracting student attention. This is an increasingly complex task, especially in today's society, in which technology is seen as a central axis. Faced with a generation that experiences a well-established "technological revolution", Augmented Reality (AR) seems to be an efficient tool to promote the inclusion of ludic elements during the learning process. The purpose of this article is to evaluate the use of AR tools such as Flaras and Aumentaty tools as resources for expositive lectures considering teachers acceptance for this practice. This work has shown some indications that AR applications can be used by any teacher who wants to explore them in the classroom and learn about them if she receives the necessary support.
\end{abstract}

Keywords: augmented reality, teaching, Aumentaty.

\section{INTRODUÇÃO}

Desde os primórdios da humanidade, aprendizado e evolução encontram-se diretamente relacionados à capacidade dos homens para interagirem com a realidade na qual estão inseridos. Inicialmente o ser humano lidava com animais e pessoas próximas. Com o passar do tempo, passou a produzir ferramentas capazes de ampliar a forma como ele interagia com o contexto ao seu redor. Mais adiante, a tecnologia se desenvolve de 
forma exponencial e os homens vão sendo cada vez mais obrigados a interagir com a máquina.

Segundo Tori et al. (2006), a Realidade Virtual (RV) “... é uma técnica avançada de interface, onde o usuário pode realizar imersão, navegação e interação em um ambiente sintético tridimensional gerado por computador, utilizando canais multi-sensoriais". Um ponto extremamente positivo para este tipo de interface é a capacidade de transferência do conhecimento intuitivo do usuário a respeito do mundo físico para manipular o mundo virtual. Dentro do escopo da RV encontra-se a Realidade Aumentada (RA), que é a área principal em que se insere este trabalho.

A RA é uma derivação da RV caracterizada pela combinação propiciada por um ambiente real sobreposto por elementos virtuais. Suas aplicações estão presentes nos mais diversos contextos da sociedade, a exemplo de pesquisas envolvendo planejamento cirúrgico, na área de entretenimento, na educação, dentre outros. Nessa perspectiva, pergunta-se: Como essa tecnologia tem sido utilizada em sala de aula? Algumas pesquisas (Chaves, 1999; Tori, 2010; Almeida e Santos, 2015) evidenciam que a tecnologia traz inúmeros benefícios para a educação, podendo ser utilizada para complementar e melhorar as aulas tradicionais, além de dar suporte à educação formal sob três perspectivas: suporte ao ensino presencial; suporte ao ensino a distância e apoio à autoaprendizagem.

Algumas pesquisas tratam especificamente sobre o uso de RA na educação, como o trabalho de Pereira et al. (2017), que apresenta um aplicativo para Android denominado Educar-Quadrics destinado ao ensino de cálculo em nível de graduação e o de Araújo et al. (2017) que descreve um estudo de caso sobre a utilização de recursos de realidade aumentada no ensino de ciências biológicas utilizando smartphones. Nos dois trabalhos a validação é feita por meio de questionários aplicados com discentes e em ambos se observou boa aceitação das propostas, mas também a necessidade de melhorias e aprofundamentos para uma melhor utilização das aplicações de RA na educação.

Considerando que se espera ver as aplicações de RA propostas em diversos trabalhos na literatura de fato chegarem às salas de aula, faz-se necessário investigar a aceitabilidade deste recurso por parte dos docentes e quão viável seu uso pode ser, já que o papel do docente nesse processo é fundamental.

Motivados pelo potencial da utilização da RA para melhoria dos processos de ensino-aprendizagem e observando no dia a dia de algumas escolas a resistência de docentes de Ensino Básico quanto ao uso da tecnologia em sala de aula, este trabalho visa investigar se esse uso é viável sob o ponto de vista do professor que por vezes vê recursos como a RA como um empecilho ao invés de um facilitador. Desse modo, o objetivo geral deste artigo é analisar a viabilidade de uso de algumas ferramentas de RA por docentes em aulas expositivas e sua aceitação. Para isto, o presente trabalho se focou nas ferramentas Flaras e Aumentaty, pelo fato de vários trabalhos da literatura tratarem delas. Como objetivos específicos deste trabalho, pode-se destacar a análise destas ferramentas e a proposição de algumas sequências didáticas explorando RA em algumas disciplinas e sua avaliação por professores.

As demais seções deste trabalho estão organizadas conforme descrito a seguir. A Seção 2 descreve os materiais e métodos utilizados. A Seção 3 apresenta e discute alguns resultados deste trabalho. Por fim, a Seção 4 apresenta as principais conclusões e propostas de trabalhos futuros. 


\section{MATERIAIS E MÉTODOS}

O presente estudo do ponto de vista empírico pode ser classificado como uma pesquisa-ação devido a sua associação com a resolução de um problema coletivo e no qual os participantes representativos do problema estão envolvidos de forma cooperativa ou participativa (Gil, 2002).

Neste trabalho se pretende investigar junto a professores do ensino básico a aceitação do uso de RA em aulas expositivas, coletando por meio de questionário específico, pontos positivos e negativos da metodologia de ensino proposta e analisandose sua aceitação da tecnologia nesse processo, considerando-se que muitas vezes há uma resistência no seu uso por alguns docentes. A introdução de uma nova tecnologia é citada por Thiollent (2011) como um exemplo de ação. Este autor destaca ainda que na ação se pretende aumentar o conhecimento dos pesquisadores e conhecimento ou "nível de consciência" das pessoas e grupos considerados. Por meio da exposição dos docentes que participaram da pesquisa aos recursos da RA que poderiam explorar se pretendia aumentar sua consciência sobre esta utilização e conhecer melhor suas percepções.

Para atingir os objetivos pretendidos, a metodologia deste trabalho incluiu os seguintes passos: Foram levantados trabalhos da literatura sobre a RA na educação; Foram analisadas de forma comparativa as ferramentas Flaras e Aumentaty para escolher uma delas para análise por docentes; Foram elaborados e apresentados a professores exemplos de sequências didáticas de algumas disciplinas (História, Biologia e Química) do ensino básico utilizando a ferramenta Aumentaty; Foi realizada a análise da viabilidade da utilização de ferramentas de RA no Ensino Básico através de questionário aplicado com professores das disciplinas tomadas como exemplo nessa pesquisa; Foram tecidas conclusões com base nas análises realizadas.

Considerando a revisão da literatura, observou-se que há vários trabalhos (Pereira, 2014; Almeida e Santos, 2015; França, 2015; Santos Júnior, 2017; Pereira et al., 2017) voltados à utilização da RA para fins educacionais envolvendo o ensino da matemática. Diante dessa realidade, optamos pela escolha de disciplinas consideradas pouco elencadas durante as pesquisas bibliográficas relativas ao tema para fazer as avaliações com professores como forma de buscar estimular o uso de RA em outras disciplinas além da matemática. Foram escolhidas para este trabalho as disciplinas de História (abordando o tema do antigo Egito com representações 3D das pirâmides e Rio Nilo), Biologia (abordando o tema estrutura celular com modelos 3D de Célula Animal e Vegetal) e Química (abordando o tema modelos atômicos, com a respectiva estrutura 3D) como está ilustrado pela Figura 1.

Foram então analisados os softwares Aumentaty e Flaras para tentar identificar qual destes poderia ser melhor aceito por professores e se optou pelo Aumentaty, que embora utilizado com certa frequência, principalmente no meio acadêmico, ainda é bastante desconhecido para grande parcela dos profissionais da educação básica e se mostrou mais adequado para o estudo com os professores, conforme detalhado a seguir. Utilizando o Aumentaty, foram elaboradas sequências didáticas' e tutoriais².

Observando-se que para a utilização de softwares dessa natureza são necessários objetos 3D e que nem sempre é fácil produzi-los, buscou-se explorar nas sequências didáticas modelos obtidos através de download em repositórios presentes na Internet. Nesse trabalho, os modelos 3D utilizados foram retirados do site 3D Warehouse $e^{3}$, uma plataforma desenvolvida pelo Google e adquirida pela Trimble em 2012, responsável

1 https://www.dropbox.com/s/436f1faehza76zf/sequenciasDidaticas.pdf?dl=0

2 https://goo.gl/zpCi9C

3 https://3dwarehouse.sketchup.com

V. $16 \mathrm{~N}^{\mathrm{o}} 1$, julho, 2018 


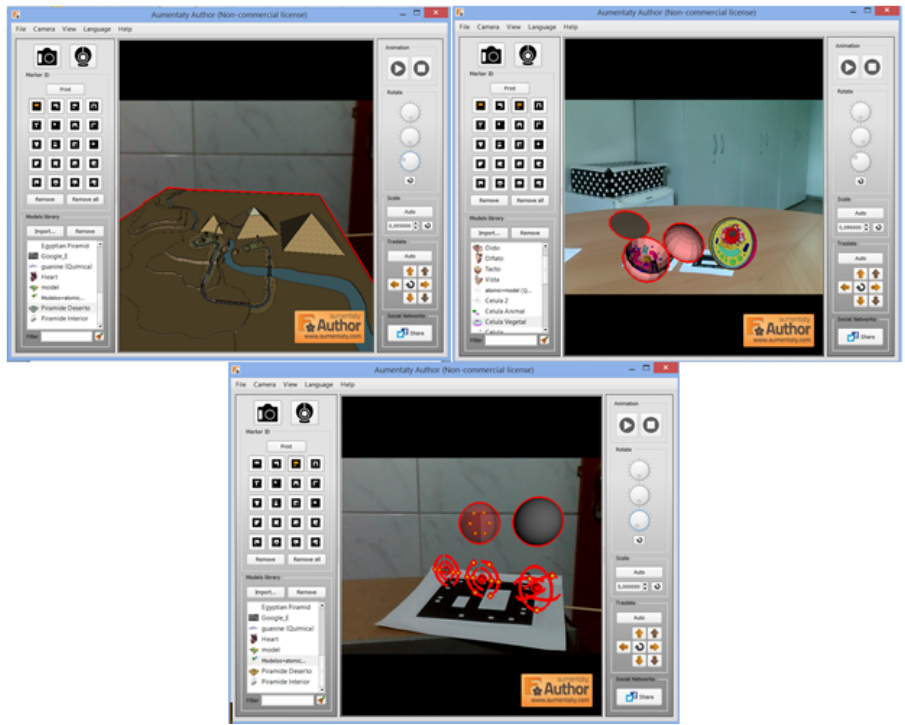

Figura 1 - Alguns dos Modelos 3D utilizados nas sequências didáticas.

por compartilhar gratuitamente diversos modelos 3D projetados por usuários das mais diversas partes do mundo.

Durante a análise do Aumentaty e Flaras foram feitos testes com alguns modelos utilizando uma máquina com a seguinte configuração:

$>$ Processador Intel ${ }^{\circledR}$ Core $^{\mathrm{TM}}$ I7-4700MQ CPU @ 2.40GHz;

$>$ Memória instalada (RAM): 32,0 GB;

$>$ Placa de Vídeo: AMD Radeon ${ }^{\mathrm{TM}}$ HD8970M 4,0 GB;

$>$ Sistema Operacional: Windows 8.1 Professional.

$>$ Webcam Chicony 5M USB

Para os testes, basicamente foi utilizada uma mesa onde eram posicionados os marcadores no momento de realizar as aplicações, além de um notebook e sua webcam. A iluminação não recebeu nenhum tratamento especial, sendo composta apenas por lâmpada de $60 \mathrm{w}$ e luz ambiente.

Para avaliar a utilização da RA por meio das sequências didáticas e tutoriais propostos, foi elaborado e submetido (através do Formulário do Google) inicialmente a professores de diversas escolas do Ensino Básico um Questionário. Esse questionário foi divulgado através da Internet (Facebook, Whatsapp, E-mail) com diversos professores do Ensino Básico. Ele tinha como objetivo apreender o conhecimento prévio que os docentes por ventura teriam ou não em relação ao tema proposto. Por exemplo, se buscou investigar se os mesmos já realizavam de alguma forma a utilização de aplicativos que acessassem o rastreamento ótico da câmera de um dispositivo móvel (ex: uso de QRCode, Whatsapp Web), algo necessário quando se explora RA. E por fim, verificou-se se conheciam de algum modo ferramentas utilizadas para gerar aplicações com RA.

Posteriormente foram elaboradas três sequências didáticas para as disciplinas escolhidas explorando a utilização de RA. Foi realizada para isso uma análise com as peculiaridades de cada um dos dois softwares de RA selecionados para este estudo: o Flaras e o Aumentaty. Durante os testes com ambos, o Flaras apresentou limitações para trabalhar com qualidade com os modelos que estavam sendo selecionados para as sequências didáticas, sendo por isso sugerido o uso do Aumentaty nas sequências didáticas utilizadas para avaliação pelos professores.

Tal avaliação foi feita por meio da aplicação de outro questionário com professores atuantes no ensino básico nas disciplinas alvo das sequências didáticas 
propostas nesse estudo e que tinham respondido o primeiro questionário. Para isso, foi realizado inicialmente o contato através de e-mail, WhatsApp ou mesmo ligação convencional, através do qual foi agendada uma visita. Nesta visita, detalhou-se a proposta pertinente à área de cada professor, demonstrando a sequência didática em funcionamento e buscando especificar como desenvolvê-la através do software Aumentaty.

Ao término da explanação, disponibilizou-se o endereço do segundo questionário online para cada um apresentar sua resposta. Isso foi feito como forma de deixar cada participante mais livre para expor sua opinião, podendo refletir com calma sobre as suas respostas. As perguntas dos dois questionários e as respostas obtidas no segundo estão disponíveis online ${ }^{4}$.

\section{RESULTADOS E DISCUSSÃO}

Nesta seção são apresentados os principais resultados obtidos em diferentes fases desta pesquisa.

\subsection{Levantamento de dados sobre o uso de RA por docentes}

O primeiro Questionário foi respondido por 33 docentes do Ensino Básico e gerou os seguintes resultados: a maioria $(54,5 \%)$ dos participantes da pesquisa tem entre $20 \mathrm{e}$ 30 anos e $42,4 \%$ apresentam entre 31 e 40 anos, estando apenas um participante na faixa de idade superior a 40 anos. $24(72,7 \%)$ deles atuam efetivamente na Educação Básica, sendo que mesmo os que não estão atuando no momento, já foram professores nessa etapa educacional. Concernente à área de atuação, a maior parcela de participantes da pesquisa foi de professores de Matemática (8), seguido por professores de Biologia (6), de História (5), de Língua Estrangeira (4), de Química (3), de Português (3), de Geografia (2) e Física (2).

Os participantes são docentes que desenvolvem suas atividades no ensino básico das seguintes cidades: Campina Grande (PB), Curitiba (PR), Itaporanga (PB), Cacimba de Dentro (PB), Ingá (PB), Itaporanga (PB), João Pessoa (PB), Lagoa Seca (PB), Livramento (PB), Parelhas (RN) e Tenório (PB).

Percebe-se que, entre o público pesquisado, a maioria (25) já teve contato com o Whatsapp Web, mostrando que eles não são tão alheios à utilização das tecnologias. Considerando o uso de QR Code, o número de conhecedores foi de 20, algo de certa forma contraditório, uma vez que para utilizar o WhatsApp Web é necessário ler o QR Code. Algo passível de suposição é que as respostas afirmativas para os que relataram utilizar Whatsapp Web, na verdade podem representar a utilização do aplicativo WhatsApp convencional ao invés do Web.

As questões relacionadas no parágrafo anterior, quanto à utilização de Whatsapp $W e b$ e QR Code, demonstram que a maioria dos professores $(75,75 \%$ no caso do Whatsapp Web e 60,60\% no caso do QR Code) já executaram uma proposta muito similar com aquilo que é necessário para implementar a RA, isto é, colocar uma câmera em frente a uma determinada figura de forma que essa câmera realize o reconhecimento da mesma. Pode-se observar que, embora possa parecer algo distante da realidade deles, como aparentemente demonstraram as questões posteriores, na prática o uso da tecnologia pode ser mais simples do que parece. 
Em se tratando da utilização da RA, é notória a atitude leiga do público estudado com relação a esse tipo de tecnologia, visto que apenas 3 pessoas declararam que já tiveram contato com o contexto. Ao serem questionados quanto ao nível de conhecimento sobre a RA, 20 entrevistados marcaram a opção "Nenhum", enquanto que 13 deles têm alguma noção e nenhum declarou conhecer bem. Ao aumentarmos o nível de especificidade das perguntas, o número de respostas positivas se mostrou mais reduzido: 2 deles declararam conhecer alguma ferramenta de RA, totalizando cerca de $6 \%$ dos entrevistados. Pediu-se para que citassem as ferramentas conhecidas e as seguintes respostas foram obtidas: Infográficos no suporte pedagógico da editora FTD e óculos simulador 3D para celular. Isto evidencia que as respostas não se referem exatamente a ferramentas que realizam aplicações com RA, uma vez que os óculos são um item de hardware responsável por sua visualização e os infográficos se tratam de uma aplicação pré-estabelecida, conforme explicação dada no site da editora FTD.

Os entrevistados também foram questionados diretamente sobre o conhecimento das ferramentas Flaras e Aumentaty, porém nenhum deles as conhecia.

\subsection{Análise das Ferramentas Aumentaty e Flaras}

Enquanto eram coletadas as respostas ao primeiro questionário, foi realizada a elaboração das sequências didáticas e o processo de testes com o auxílio do software de RA Aumentaty e do Flaras.

Embora França (2015) tenha defendido a ideia que o Flaras apresenta interface bastante simples e acessível a qualquer usuário, além de ser de extrema utilidade para implementação de aplicações de RA, a experiência obtida ao testar seu uso com os modelos utilizados nas sequências didáticas propostas demonstrou que o programa mostrou-se incapaz de rodar as aplicações. É importante destacar que os modelos utilizados diferiam da maioria das demonstradas pelo autor citado acima e onde se explorava objetos mais simples.

Outro trabalho mais recente (Santos Junior, 2017) já demonstra que o Flaras se mostrou muito limitado. $\mathrm{O}$ autor pontua que a interface do software exige uma alta curva de aprendizado, não sendo, portanto muito intuitivo. Além disso, sugere que o aplicativo foi incapaz de visualizar mais de uma estrutura simultaneamente e não conseguiu trabalhar com cores distintas em um mesmo objeto.

A experiência obtida nos testes comparativos realizados no presente trabalho corrobora com a maioria das inferências de Santos Junior (2017), uma vez que a ferramenta apresentava problemas ao gerar as aplicações sugeridas pelas sequências didáticas elaboradas.

No entanto, percebe-se que na maioria dos casos mencionados pelos trabalhos elencados anteriormente, em que o Flaras foi utilizado de modo satisfatório, as aplicações limitavam-se a estruturas geométricas simples. Isso evidencia que alguns pesquisadores podem não ter encontrado problemas como os descritos nesta seção pelo baixo nível de complexidade dos objetos 3D utilizados. Pode-se observar que apenas em uma das sequências, a de Química, o Flaras foi capaz de rodar a aplicação do modelo atômico de modo que fosse possível distinguir minimamente as formas. Verificando estes aspectos, concluiu-se que não seria recomendada sua utilização em uma aula expositiva, pois a representação gráfica estava longe de condições satisfatórias para tal. Salienta-se que todos os arquivos estavam no formato *.kmz, suportado pelo programa, e as configurações da máquina que rodaram todas as aplicações deste trabalho eram mais que suficientes para prover o suporte de processamento que os programas necessitam. 
Observou-se também pela experiência com as sequências didáticas propostas que a interface do Flaras não se mostrou muito amigável. Outras limitações percebidas referem-se ao fato do programa não permitir a fixação de objetos na tela, tornando dispensável o uso do marcador, o que pode ser um grande problema numa aula expositiva, na medida em que alterações no posicionamento da câmera muitas vezes involuntárias refletem diretamente na geração dos objetos em RA.

Com base nos testes realizados durante a execução desse trabalho, bem como em outras pesquisas envolvendo a ferramenta, como a de Pereira (2014) e Santos Júnior (2017), observa-se que seria mais interessante na análise junto aos professores explorar outra ferramenta ao invés do Flaras, embora possa ser utilizado para rodar aplicações mais simples como as descritas no trabalho de Pereira (2014) e em outros trabalhos que exploram essa ferramenta na área de geometria.

Considerando o que Almeida e Santos (2015) e Santos Júnior (2017) relataram a respeito do uso da ferramenta Aumentaty e o ganho significativo no alcance do propósito educacional utilizando RA, optou-se por este software, mas buscando explorar outras disciplinas além da matemática para complementar a literatura com trabalhos explorando RA em outras disciplinas e investigando a visão de professores de outras áreas.

Foram realizadas tentativas de implementar inicialmente as mesmas sequências didáticas nos dois softwares. Porém apenas um deles, Aumentaty, obteve êxito, enquanto o Flaras conseguiu apenas a aplicação com modelos atômicos, mas com imperfeições que gerariam um impacto negativo a respeito do uso da RA para trabalhar os conteúdos programados. Ao término dos testes iniciais das sequências didáticas com os referidos softwares, foi possível elaborar o Quadro 1, apresentado a seguir, que representa as impressões obtidas pelos autores após estes testes.

Quadro 1 - Comparativo entre os Softwares de RA Flaras 2.4.3 x Aumentaty 1.3. considerando as impressões obtidas após os testes realizados neste trabalho

\begin{tabular}{|c|c|c|}
\hline $\begin{array}{l}\text { Software } \\
\text { Parâmetro }\end{array}$ & Flaras & Aumentaty \\
\hline Formatos Aceitos & $\begin{array}{l}\text { Objetos 3D: DAE/3DS zipped } \\
\text { Files (.zip) ou Files (.kmz); } \\
\text { Imagens/Vídeos: .jpg, .gif, .png, } \\
\text {.mp4, .flv. }\end{array}$ & $\begin{array}{l}\text { Objetos 3D: .3ds, .fbx, .obj, .dae; } \\
\text { Imagens/Vídeos: .png, .jpg, .bmp, . } \\
\text { mpg, .avi, .mp4 }\end{array}$ \\
\hline $\begin{array}{c}\text { Percepção dos } \\
\text { autores sobre a } \\
\text { Facilidade de Uso }\end{array}$ & $\begin{array}{l}\text { Requer um maior nível de } \\
\text { intimidade com a interface para } \\
\text { realizar aplicações de RA. }\end{array}$ & $\begin{array}{l}\text { Intuitiva, parece ser de fácil acesso } \\
\text { para usuários leigos de um modo } \\
\text { geral. }\end{array}$ \\
\hline $\begin{array}{l}\text { Qualidade de } \\
\text { Exibição dos } \\
\text { Objetos }\end{array}$ & $\begin{array}{l}\text { Exibe alguns objetos de forma } \\
\text { satisfatória. No entanto, } \\
\text { conforme aumenta a } \\
\text { complexidade dos objetos a } \\
\text { serem exibidos, menor será a } \\
\text { eficácia do programa para exibi- } \\
\text { los. }\end{array}$ & $\begin{array}{l}\text { Apresenta um desempenho bastante } \\
\text { elevado em relação ao Flaras, tanto } \\
\text { na qualidade de exibição dos } \\
\text { arquivos 3D, quanto na velocidade } \\
\text { de processamento. }\end{array}$ \\
\hline $\begin{array}{c}\text { Trabalha com } \\
\text { Objetos } \\
\text { Simultaneamente }\end{array}$ & $\begin{array}{l}\text { Muitas vezes apresenta } \\
\text { dificuldades para trabalhar com } \\
\text { objetos que demandam maior } \\
\text { capacidade de processamento, } \\
\text { por isso não é recomendável } \\
\text { nesses casos. }\end{array}$ & $\begin{array}{l}\text { Sim, o que se torna um recurso bem } \\
\text { útil para o enriquecimento do } \\
\text { processo didático. }\end{array}$ \\
\hline
\end{tabular}




\begin{tabular}{|l|l|l|}
\hline $\begin{array}{c}\text { Dependência do } \\
\text { Marcador }\end{array}$ & $\begin{array}{l}\text { Necessita do marcador para gerar } \\
\text { e manipular o objeto 3D do início } \\
\text { ao fim da aplicação. }\end{array}$ & $\begin{array}{l}\text { Uma vez gerado o modelo 3D na } \\
\text { tela, pode dispensar a utilização do } \\
\text { marcador. }\end{array}$ \\
\hline \multirow{5}{*}{ Conceito Final } & $\begin{array}{l}\text { Atende às expectativas para } \\
\text { aplicações mais simples. No }\end{array}$ & $\begin{array}{l}\text { O Aumentaty se mostrou uma } \\
\text { ferramenta mais robusta e superou o }\end{array}$ \\
entanto, não alcança muitas & Flaras em diversos aspectos tais \\
& $\begin{array}{l}\text { vezes um nível atrativo em } \\
\text { termos de recursos relativos à } \\
\text { trabalhados numa mesma aplicação; } \\
\text { facilidade de uso (avaliada } \\
\text { informalmente) para conquistar }\end{array}$ & $\begin{array}{l}\text { Qualidade de exibição dos Objetos } \\
\text { 3D; Facilidade de utilização. }\end{array}$ \\
\hline $\begin{array}{l}\text { o usuário ou mesmo em aspectos } \\
\text { qualitativos para a geração dos } \\
\text { objetos em RA. }\end{array}$ & \\
\hline
\end{tabular}

\subsection{Avaliação das Sequências Didáticas e do uso de RA por Docentes}

Como forma de investigar a viabilidade da proposta de utilização da RA em aulas expositivas no Ensino Básico, as sequências didáticas propostas e implementadas em RA através do Aumentaty foram apresentadas para três professores que atuam na Educação Básica, através de visita in loco, sendo um de cada uma das disciplinas tomadas como objetos do estudo. As idades dos professores que responderam a avaliação são as seguintes: o de Biologia (P1) tem 32 anos, o de História (P2) tem 28 anos e o de Química (P3) tem 34 anos. Em momento posterior, foi aplicado o questionário de avaliação da metodologia proposta. Dois deles (P1 e P2) ministram aulas há 3 anos, enquanto o outro (P3) tem 14 anos de experiência no Ensino Básico. Dentre os três, dois (P1 e P3) tiveram experiências tanto no Ensino Fundamental quanto no Ensino Médio, enquanto o outro (P2) apenas no Ensino Médio.

No que se refere ao nível de contribuição da RA para o ensino, os professores demonstraram-se entusiasmados com a proposta, e fizeram algumas observações importantes nesse sentido. Alguns aspectos destacados foram os seguintes: A possibilidade de explorar conteúdos de forma aprofundada, em especial aqueles que necessitam de análises de estruturas, espaços geográficos, monumentos e edifícios (P2); A maneira como pode encantar os estudantes (P2); Maior sensação de interatividade (P1); Capacidade para inserir o 3D na explanação de conteúdos através dos quais somente era possível utilizar o plano XY (P3).

Quanto à utilização da ferramenta de um modo geral, não demonstraram grandes dificuldades para lidar com ela, visto que as respostas evidenciaram que, no máximo, o grau de dificuldade para lidar com o software era moderado, algo indicado apenas por P3 enquanto P1 e P2 consideraram a ferramenta Aumentaty fácil de ser utilizada.

$\mathrm{Na}$ questão inerente à receptividade da metodologia por parte dos professores em geral, segundo a opinião dos participantes, verificou-se que dos três, dois (P2 e P3) responderam "Talvez". Foram feitas as seguintes justificativas às respostas: apego excessivo a práticas de ensino tradicionais (P2); dificuldade para lidar com novas tecnologias e/ou linguagens (P2); além de ser essa uma questão de cunho pessoal, pois enquanto muitos docentes poderiam aderir à utilização sem grande resistência, com certeza existe outra parcela que lidaria com bastante resistência à aceitação dessa novidade (P3). Aquele que respondeu "Sim" (P1) indicando que achava que a metodologia seria bem aceita, justificou da seguinte forma: "Quando os professores tiverem acesso ao material relacionado a como utilizar a ferramenta e perceberem que existe certa facilidade em relação ao seu uso, não haverá razões que impeçam qualquer profissional da educação de se valer dessa tecnologia, quando necessário, pois ela abre 
margem para muitas opções capazes de dinamizar e melhorar o ensino".

Ainda sobre os resultados do segundo questionário, foi possível perceber indícios de que mesmo para professores que tinham a RA como algo distante de seu cotidiano foi possível perceber a sua receptividade por meio da experiência com o Aumentaty. Viu-se também que pode ser viável o uso dessa tecnologia no âmbito educacional com esta ou outras ferramentas, mas que é preciso vencer de alguma forma a resistência que alguns docentes podem apresentar.

Apesar do grande potencial existente para o uso da RA na educação, é imprescindível buscar estratégias para que a adoção do uso se dê de maneira tranquila para o professor, explorando ferramentas que se mostrem adequadas para o que ele deseja trabalhar, e fazendo com que este se torne ciente dos vários recursos já prontos do qual pode se beneficiar para tornar suas aulas mais dinâmicas, como os vários modelos 3D disponíveis na Web e as diferentes ferramentas de RA. Neste sentido, o apoio de profissionais da área de tecnologias educacionais é fundamental para que se possa vencer os diferentes obstáculos encontrados no contexto escolar para que o uso de recursos como a RA se torne uma realidade nas escolas e o aprendizado para os alunos se torne algo mais prazeroso e dinâmico.

\section{CONCLUSÕES E TRABALHOS FUTUROS}

Este trabalho teve como objetivo analisar a viabilidade do uso de ferramentas de RA como o Flaras e o Aumentaty como recursos para aulas expositivas considerando sua aceitação por professores. Observou-se que por meio do presente trabalho foi possível levar ao conhecimento de docentes do Ensino Básico uma proposta de utilização da RA em aulas expositivas e que estes se mostraram receptivos com a proposta, elencando diversos pontos positivos após terem um contato com sequências didáticas de suas áreas explorando esse recurso.

No entanto, viu-se por meio do estudo que pode haver resistência por parte de alguns docentes quanto ao uso da RA em aulas, mas talvez dependendo da forma de apresentar a proposta, e do apoio que o docente pode receber, pode ser possível trazer para os alunos aulas bem diferentes das que costumam ter.

Acredita-se que o presente trabalho deu indícios de que é viável de fato usar ferramentas de RA em aulas expositivas, mas é preciso investigar se as ferramentas são adequadas para os modelos 3D que se pretende trabalhar, pois como se viu nos testes com o Flaras, algumas ferramentas podem apresentar problemas, e dificultar ainda mais a aceitação do uso da técnica de RA por parte dos docentes. Além disso, observou-se que o apoio técnico-pedagógico ao docente pode ser um fator a contribuir substancialmente na aceitação das novas práticas pedagógicas apoiadas pela tecnologia.

De qualquer forma, acredita-se que uma contribuição deste trabalho é incentivar a exploração por docentes em suas aulas de modelos existentes como os do 3D Warehouse através de ferramentas de RA como o Aumentaty. Como trabalhos futuros, espera-se fazer melhorias e divulgar os tutoriais já preparados para facilitar o uso de ferramentas de RA por docentes e preparar novos tutoriais considerando também outras ferramentas, além de diferentes sequências didáticas que possam favorecer a adoção da prática por professores de diferentes áreas. Espera-se também no futuro poder observar os resultados da aplicação na prática destas sequências didáticas propostas e coletar as impressões de mais professores. 


\section{AGRADECIMENTOS}

Gostaríamos de agradecer a todos os professores que participaram da pesquisa respondendo aos questionários, bem como aos professores Eudisley Gomes dos Anjos e Josilene Aires Moreira pelas sugestões dadas a este trabalho.

\section{REFERÊNCIAS BIBLIOGRÁFICAS}

ALMEIDA, M. L. de; SANTOS, G. Realidade aumentada na educação. Revista Tecnologias na Educação, ano 7, v. 12. ISSN 1984-4751 [S.I], jul. 2015. Disponível em: < http://tecedu.pro.br/wp-content/uploads/2015/07/Art2-vol12-julho2015.pdf>. Acesso em jun. 2017.

ARAÚJO, L.; MACHADO, M. B.; VASCONCELlos, A. P.; TAVARES, T. A. DoctorBio: Um Estudo de Caso sobre a Utilização de Recursos de Realidade Aumentada no Ensino de Ciências Biológicas. In: Anais do Workshop de Informática na Escola (WIE 2017). 2017.

CHAVES, E. O. C. Tecnologia na educação, ensino a distância e aprendizagem mediada pela tecnologia: conceituação básica. Revista de Educação, PUC - Campinas, v. 3, n. 7, p, 29-43, nov. 1999. Disponível em: <http://periodicos.puccampinas.edu.br/seer/index.php/reveducacao/article/view/421>. Acesso em maio. 2017.

FRANÇA, J. S. Uma proposta didática da realidade aumentada no ensino da geometria espacial. Universidade Federal do Pará. Belém, p. 54. 2015. (ISBN).

GIL, A. C. Como elaborar projetos de pesquisa. 4. ed. São Paulo : Atlas, 2002.

SANTOS JUNIOR, R. J. dos. Utilizando a realidade aumentada em uma metodologia para o ensino de geometria espacial voltada ao ensino básico. Universidade Estadual da Paraíba. Campina Grande, p. 63. 2017.

PEREIRA, D. D. Uso de realidade aumentada como ferramenta de apoio ao ensino da geometria espacial no $2^{\circ}$ ano do ensino médio: utilização das ferramentas Flaras e Google Sketchup. Universidade Estadual da Paraíba. Campina Grande, p. 51. 2014.

PEREIRA, L. T.; OLIVEIRA, D. C. B.; COUTO, I. F.; OLIVEIRA, A. M.; SILVA, R. L. S. Uma ferramenta de Apoio ao Ensino de Calculo com Realidade Aumentada. In: Anais do Simpósio Brasileiro de Informática na Educação. 2017.

TORI, R. A presença das tecnologias interativas na educação. Revista de Computação e Tecnologia da PUC-SP - Departamento de Computação/FCET/PUC. v. II, n. 1, p. 4-16, 2010. Disponível em: <https://revistas.pucsp.br/index.php/ReCET/article/view/3850>. Acesso em maio. 2017.

TORI, R.; KIRNER, C.; SISCOUTTO, R. Fundamentos e tecnologia de realidade virtual e aumentada. Porto Alegre: SBC - Sociedade Brasileira de Computação, 2006.

THIOLLENT, M. Metodologia da pesquisa-ação. 18. ed. São Paulo: Cortez, 2011. 